\title{
Posterior ocular structural and vascular alterations in severe COVID-19 patients
}

\author{
Nurettin Bayram ${ }^{1}$ (D) $\cdot$ Medine Gundogan ${ }^{1} \cdot$ Cemal Ozsaygılı ${ }^{1} \cdot$ Ron A. Adelman ${ }^{2}$
}

Received: 4 June 2021 / Revised: 27 August 2021 / Accepted: 19 September 2021 / Published online: 4 October 2021

(c) The Author(s), under exclusive licence to Springer-Verlag GmbH Germany, part of Springer Nature 2021

\begin{abstract}
Purpose This study aimed to evaluate posterior ocular structural and vascular changes in severe coronavirus disease 2019 (COVID-19) patients.

Methods This was an observational, prospective, and controlled study including 106 eyes of 53 severe COVID-19 patients, compared to after recovery and 106 eyes of 53 age- and gender-matched healthy controls. All subjects were previously healthy adults and were assessed using spectral domain optical coherence tomography (SD-OCT) and ImageJ software. Subfoveal over a $1500-\mu \mathrm{m}$ span and macular over a $6000-\mu \mathrm{m}$ span cross-sectional areas of the vascular, stromal, and total choroid were measured. Results Of the 53 included patients, 28 (52.8\%) were male, and 25 (47.2\%) were female, with a mean age of $50.2 \pm 7.4$ years. In the active period of the disease, compared to after recovery and healthy controls, the outer plexiform layer thickness showed a significant increase $(p=0.004)$, and mean choroidal thickness was significantly higher $(p<0.0001)$; however, choroidal vascularity was significantly lower $(p<0.0001)$. The stromal area to vascular area $(\mathrm{S} / \mathrm{V})$ ratio of the choroid was significantly increased $(p<0.0001)$. All quadrants of the peripapillary retinal nerve fiber layer (RNFL) thicknesses were significantly increased (for all, $p<0.05$ ). The reflectivity of OCT echo of the choroid and peripapillary RNFL was significantly higher ( $p=0.023, p<0.0001$, respectively). Conclusion This study detected significant posterior ocular structural and vascular alterations in patients with severe COVID19 infections. These findings may be associated with direct host-virus interaction or linked to an autoimmune process, vasculopathy, or viral-mediated inflammation.
\end{abstract}

Keywords Coronavirus disease 2019 - Posterior ocular structures · Optical coherence tomography · Choroidal vascularity . Reflectivity of OCT echo

\section{Key messages}

- A few studies have reported posterior ocular structural changes in patients recovering from COVID-19. However, there has been no study evaluating posterior ocular structural and vascularity changes in the active phase of COVID-19, compared to post-recovery and healthy controls.

- This study shows that the outer plexiform layer, choroidal thickness, total choroidal area and all quadrants of the peripapillary RNFL, along with the reflectivity of OCT echoes of the choroid and peripapillary RNFL, were higher in the active period of the disease.

- The choroidal vascularity index was lower.

Nurettin Bayram

drnbayram@gmail.com

1 Department of Ophthalmology, Kayseri City Training and Research Hospital, University of Health Science, 38080 Kayseri, Turkey

2 Department of Ophthalmology and Visual Science, Yale University School of Medicine, New Haven, CT, USA

\section{Introduction}

The coronavirus disease 2019 (COVID-19), caused by the severe acute respiratory syndrome coronavirus 2 (SARSCoV-2), leads to multi-system dysfunction with many pathways that have emerged as a mediator of severe disease. One of these pathways is disease-related vascular 
system dysfunction. The SARS-CoV-2 causes a vascular system injury through immune dysregulation, hyperinflammatory process, endothelial cell dysfunctions, and hemodynamic instability [1]. Severe COVID-19 patients reveal the immune system's activation that presents cytokine storm, macrophage activation syndrome, and subsequent immune dysregulation [2]. The hyper-inflammatory state results from an acute increase in circulating levels of various pro-inflammatory cytokines, including interleukin (IL)-1, IL-6, interferon- $\gamma$, and tumor necrosis factor- $\alpha[1,2]$. Inflammation in the vascular system can cause diffuse microangiopathies and thrombosis.

A previous study detected SARS-CoV-2 nucleic acid in the human retina in deceased COVID-19 patients [3]. Clinical studies from COVID-19 patients reveal retinal microangiopathy, presenting with cotton wool retinal exudates $[4,5]$. Increased retinal vessel diameters, tortuous vessels, and retinal hemorrhages were also reported [5].

Posterior ocular structures are susceptible to many pathologies, including systemic inflammation [6]. Since SARS-CoV-2 infection causes systemic hyper-inflammation and vascular system injury, exploring and measuring the posterior ocular structure as a potential indicator is essential in understanding disease pathophysiology and better monitoring progression.

To the best of our knowledge, no similar study has been conducted so far on the posterior ocular structure alterations through a sample of the severe COVID-19. Herein, we present a structural analysis of the inner and outer retinal layers, peripapillary retinal nerve fiber layer (RNFL), choroidal morphological and vascular features, and the reflectivity of optical coherence tomography (OCT) echoes in a cohort of hospitalized patients positive for SARSCoV-2 during the active period of the disease, compared to after recovery and age- and gender-matched healthy controls.

\section{Materials and methods}

\section{Participants}

This prospective observational study included 106 eyes of 53 severe COVID-19 patients among 51,158 COVID19 patients hospitalized between August 2020 and April 2021, and 106 eyes of 53 age- and gender-matched healthy controls. The study was conducted at the Ophthalmology Department of Kayseri City Training and Research Hospital. The study protocol was approved by the Institutional Review Board $(\# 135,719,921)$ and conducted under the Declaration of Helsinki's tenants. Study information was given before patients' enrolment, and written informed consent was obtained from all participants.

All selected patients who were previously healthy adults, presented with severe COVID-19 and had not receive prior COVID-19 treatments, were consecutively included in the study. They were all hospitalized for respiratory support with noninvasive mechanical ventilation or high-flow nasal cannula, with a confirmed diagnosis of SARS-CoV-2 infection by a positive real-time polymerase chain reaction. Severe COVID-19 was defined as dyspnea, respiratory frequency $\geq 30$ breaths/min, a saturation of oxygen $(\mathrm{SpO} 2) \leq 93 \%$ and a ratio of the arterial partial pressure of oxygen to fraction of inspired oxygen $(\mathrm{PaO} 2 /$ $\mathrm{FiO} 2)<300 \mathrm{~mm} \mathrm{Hg}$, and/or lung infiltrates $>50 \%$ [7].

All patients underwent a comprehensive ophthalmologic examination on the day of their hospitalization, prior to any treatment being started, as well as in the third month of follow-up after recovery, which included measurement of the best-corrected visual acuity (BCVA), anterior segment examination, and a fundus examination. Refractive errors and intraocular pressure were measured with an autorefractokeratometer/tonometer (Nidek Tonoref II, Nidek Ltd, Gamagori, Japan). Axial length and anterior chamber depth measurements were obtained using optical biometry (IOLMaster 500, Carl Zeiss Meditec, Jena, Germany) by an experienced technician (AS). Automated segmentation of the macula, enhanced depth imaging (EDI) OCT, and peripapillary retinal nerve fiber layer (RNFL) were obtained with the spectral domain (SD) OCT (Spectralis HRA+OCT; Heidelberg Engineering, Heidelberg, Germany) at the same time, also by an experienced technician (SE). The patients' complaints, vital signs, and routine laboratory tests, including white blood cell (WBC), C-reactive protein (CRP), erythrocyte sedimentation rate (ESR), procalcitonin, and D-dimer levels, were recorded. Routine laboratory tests were repeated in the third month of the follow-up. The exclusion criteria included axial myopia and advanced hyperopia (spherical equivalent $>3$ diopters, or $>26 \mathrm{~mm}$ or $<21 \mathrm{~mm}$ axial length), any ocular and systemic diseases, previous ocular surgery or ocular trauma, incomplete follow-up, and poor-quality OCT images. Patients with signs and symptoms of COVID-19 or otherwise abnormal laboratory tests at the third month of follow-up, including high acute phase reactants as systemic inflammatory markers, were excluded from the study.

\section{OCT scan analysis}

Automated segmentation of the macula was obtained using the SD-OCT software automatic calculation system (version 6.0.0.2). Each set of images comprised 25 sections and 768 A-scans in a $20 \times 20^{\circ}$ rectangle encompassing the macula. Distance between consecutive scans was about $240 \mu \mathrm{m}$ and each scan was $5.8 \mathrm{~mm}$ in length. The signal-to-noise ratio was 
maximized using the automatic real-time (ART) averaging mode. Central macular thickness (CMT) was calculated on the 1-mm Early Treatment Diabetic Retinopathy Study (ETDRS) circle, centered on the fovea. The mean retinal thickness of the individual retinal layers was analyzed, including the retinal nerve fiber layer (RNFL), ganglion cell layer (GCL), inner plexiform layer (IPL), inner nuclear layer (INL), outer plexiform layer (OPL), outer nuclear layer (ONL), retinal pigment epithelium (RPE), inner retinal layers (IRLs), and outer retinal layers (ORLs). The SD-OCT macular segmentation was evaluated by two independent masked and experienced ophthalmologists (CO and MG). Before automatic measurements were taken, segmentation errors were manually corrected in the retinal layers if the segmentation lines were not correctly aligned. The analysis was performed within a 1-, 3-, and 6-mm diameter circle, centered on the fovea and evaluated in a $6 \times 6 \mathrm{~mm}$ square macular cube protocol (Fig. 1).

The choroid was imaged using a scanner superluminescent diode laser with a wavelength of $870 \mathrm{~nm}$ of the SDOCT's EDI mode after pupil dilation. A reverse image was obtained from the retina while the patient sustained fixation on the internal fixation light, and was automatically inverted for the chorioretinal interface adjacent to the zero delay. Each set of images comprised 13 sections and 768 A-scans in a $5 \times 30^{\circ}$ rectangle, encompassing the macula and optic nerve. Distance between consecutive scans was on average about $122 \mu \mathrm{m}$ and each scan was $8.8 \mathrm{~mm}$ in length. Choroidal thickness (CT) was determined as the vertical distance between the external limit of the Bruch's membrane and the choroidal-scleral interface. CT was manually measured at subfoveal and at nasal and temporal regions of $3 \mathrm{~mm}$ from the fovea center using the SD-OCT software's caliper tool (Fig. 2). The average of subfoveal, nasal, and temporal CT measurements was accepted as mean CT. All CT measurements were performed by two independent masked and experienced ophthalmologists ( $\mathrm{CO}$ and $\mathrm{MG}$ ). Inter-examiner reproducibility of all manual measurements was evaluated by the intraclass correlation coefficient (ICC) so that values of greater than 0.80 were accepted as good agreement. When the examiners' measurements were not consistent, they were repeated until good agreement was achieved and the average of both measurements was used for the final analysis.

The peripapillary RNFL thickness measurements were automatically calculated with 16 consecutive circular B-scans containing $768 \mathrm{~A}$-scans in $3.7 \mathrm{~mm}$ diameter, centered on the optic disc. The SD-OCT software provided a global average and the mean thicknesses of six regions obtained by dividing the scan circle into the global $\left(\mathrm{G}, 360^{\circ}\right)$, temporal $(\mathrm{T}$,

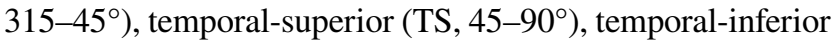
(TI, 270-315 $)$, nasal $\left(\mathrm{N}, 135-225^{\circ}\right)$, nasal-superior (NS, 90-135 ${ }^{\circ}$ ), and nasal-inferior (NI, 225-270 ${ }^{\circ}$ ) regions (Fig. 3).

For all SD-OCT images, each scan was acquired in highspeed mode and averaged 100 times during acquisition to reduce speckle noise. Each B-scan was evaluated for analysis if ART averaging $\geq 75$ times. According to signal strength, the SD-OCT images with a quality score $\geq$ of $20 \mathrm{~dB}$ (range: $0-40 \mathrm{~dB}$ ) were assessed for analysis.

Detailed ocular examinations, autorefractokeratometer/ tonometer, optical biometry, and OCT scans of all admitted patients were performed in the ophthalmology outpatient clinic, after work hours (after everyone left the eye clinic) or on the weekends. All OCT scans were obtained at a similar time interval (between 5:30 and 7:30 pm) to avoid daily physiological changes. All personnel involved in the patients' care used complete protective equipment, including an N95 mask, gown, gloves, eye protection, and face shield. After the procedures, the environment and all devices were meticulously cleaned by professional cleaning staff, based on disinfection recommendations from the American Academy of Ophthalmology [8].

\section{Choroidal vascularity index calculation}

The horizontal EDI-OCT scan passing through the fovea of each eye was used for image analysis. The images were binarized using the public domain software ImageJ (Version 1.53, National Institutes of Health, Bethesda, MD, USA; https://imagej.nih. gov/ij/) after converting to 8 bits to distinguish between the vascular and stromal areas of the choroid. The Niblack auto local threshold technique was used for image binarizations [9]. The total choroidal area, the vascular area, and the stromal area were then calculated for the macula within the central $6.0 \mathrm{~mm}$ zone and the fovea within the central $1.5 \mathrm{~mm}$ zone. Bright pixels were defined as the choroidal stromal (interstitial) area, whereas dark pixels were defined as the vascular area (Fig. 3). The choroidal vascularity index (CVI) was defined as the ratio between the vascular area and the total choroidal area.

\section{Automatic grading of OCT echogenicity}

The reflectivity of the OCT echo was automatically measured by ImageJ software. OCT scans were converted into 8-bit greyscale images. The region of interest was selected using the 'Polygon' standard drawing function (Fig. 4a-f ), and the mean value of unweighted intensity was measured using the 'Histogram' function, which determines it via the image processing software, ranging from 0 to 255 levels on the histogram panel (Fig. $4 \mathrm{~g}-1$ ).

\section{Statistical analysis}

All data were analyzed using the SPSS program for Mac OS version 26.0 (SPSS Inc., Chicago, IL, USA). Quantitative variables were expressed as the mean \pm standard deviation and categorical variables were presented as frequencies and percentages. The Kolmogorov-Smirnov test was used to determine the normal distribution of the variables. Levene's 
test was used to assess the homogeneity of variances. The differences among baseline, after recovery and healthy controls, were compared with a one-way analysis of variance for parametric data. When a significant result was obtained, the Scheffe test was performed for post hoc comparisons. Pearson's chi-square test was used for the comparison of categorical variables. The Kruskal-Wallis test was used to compare the differences in non-parametric data and we applied Bonferroni corrections in cases of significant differences. Spearman's correlation coefficient investigated the relationship between the differences of posterior ocular structures during the active period of the disease and after recovery, as well as the laboratory parameters. G* power 3.1 software was used for the power analysis. It was calculated that there should be at least 84 eyes in each group to calculate $1-\mu$ difference on SD-OCT among the groups, for 0.95 power with 0.25 effect size and $5 \%$ margin of error from 3 groups. A $p$ value less than 0.05 was considered statistical significance.

\section{Results}

Of the 71 subjects initially enrolled, 18 were excluded based on the clinical exclusion criteria: axial length $>26 \mathrm{~mm}$ $(n=1)$, ocular and/or systemic diseases $(n=8)$, poor OCT image quality $(n=2)$, previous ocular surgery, ocular trauma $(n=2)$, incomplete follow-up $(n=4)$, or any signs and symptoms or abnormal laboratory results at the 3rd month of follow-up $(n=1)$. After exclusions, 106 eyes of 53 patients with clinical, laboratory, and radiological confirmed severe COVID-19 were analyzed in this study.

Of the included patients, 28 (52.8\%) were male and 25 (47.2\%) were female, with a mean age of $50.2 \pm 7.4$ years (range: 34-65 years). The main symptoms and signs at the time of hospital admission were myalgia or fatigue in all patients (100\%), cough in 50 patients (94.3\%), dyspnea in all patients (100\%), sore throat in 41 patients $(77.3 \%)$, headache in 39 patients (73.6\%), fever in 25 patients $(47.1 \%)$, expectoration in 24 patients $(45.2 \%)$, a loss of taste and smell in 11 patients (20.7\%), and diarrhea in 7 patients (13.2\%). One of the patients $(1.9 \%)$ had conjunctival hyperemia and epiphora. Demographic features, vital signs, and laboratory results of the patients at the time of hospital admission are shown in Table 1.

The axial length and anterior chamber depth were $23.42 \pm 0.68$ and $3.42 \pm 0.49$ in the active period of the disease, $23.38 \pm 0.84$ and $3.49 \pm 0.52$ after recovery, and $23.34 \pm 0.75$ and $3.51 \pm 0.48$ in healthy controls, respectively. There were no significant differences in the axial length and anterior chamber depth in the active period of the disease, compared to after recovery and healthy controls ( $p=0.939$ and $p=0.521$, respectively). The macula, EDI, and RNFL OCT images' quality scores did not significantly
Table 1 Demographic features, vital signs, and overall inflammatory and coagulation status of the patients at hospital admission

\begin{tabular}{ll}
\hline Patient characteristics & At hospital admission \\
\hline Demographic features & \\
$\quad$ Age (years) & $50.2 \pm 7.4$ \\
Gender, male/female, $(\%)$ & $28(52.8 \%) / 25(47.2 \%$ ) \\
BCVA (Snellen chart) & $20 / 20$ \\
Intraocular pressure (mmHg) & $15.1 \pm 2.8$ \\
Axial length (mm) & $23.4 \pm 0.7$ \\
Vital signs & \\
Body temperature $\left({ }^{\circ} \mathrm{C}\right)$ & $37.5 \pm 1.1$ \\
Pulse rate (per minute) & $93.0 \pm 11.4$ \\
Respiration rate (per minute) & $32.5 \pm 1.5$ \\
SpO2 on room air $(\%)$ & $88.8 \pm 2.5$ \\
Laboratory results & \\
WBC (normal range: $\left.4.5-1010^{3} / \mu \mathrm{l}\right)$ & $6.5 \pm 2.610^{3} / \mu \mathrm{l}$ \\
CRP (normal range: $0-5 \mathrm{mg} / \mathrm{l})$ & $53.4 \pm 51.5 \mathrm{mg} / 1$ \\
ESR (normal range: $0-20 \mathrm{~mm} / \mathrm{h}$ ) & $30.7 \pm 14.0 \mathrm{~mm} / \mathrm{h}$ \\
Procalcitonin (normal value $<0.05 \mu \mathrm{g} / \mathrm{l})$ & $0.2 \pm 0.37 \mu \mathrm{g} / \mathrm{l}$ \\
D-Dimer (normal range: $220-500 \mu \mathrm{g} / \mathrm{l})$ & $829.1 \pm 859.8 \mu \mathrm{g} / 1$ \\
\hline
\end{tabular}

Abbreviations: $B C V A$ best-corrected visual acuity, $C R P$ C-reactive protein, $E S R$ erythrocyte sedimentation rate, $S p O 2$ oxygen saturation, $W B C$ white blood cell

differ among the active phase of the disease, after recovery, and healthy controls (for all, $p>0.05$, Tables 2,3 , and 4).

Although increasing CMT in the active phase of the disease is not statistically significant compared to after recovery and healthy controls $(p=0.285)$, the OPL showed a significantly higher thickness in the segmentation analysis of the central macular area $(29.6 \pm 8.8 \mu \mathrm{m}, 26.5 \pm 5.8 \mu \mathrm{m}$, and $25.8 \pm 6.3 \mu \mathrm{m}$, respectively, $p=0.004$ ) (Fig. 1a and b, ). There were no significant differences in the mean thickness of the RNFL, GCL, IPL, INL, ONL, RPE, and ORL in the active period of the disease compared to after recovery and healthy controls (for all, $p<0.05$ ). The results of the segmentation analysis of the central macular area are summarized in Table 2.

There were significant differences in choroidal characteristics in the active period of the disease, compared to after recovery and healthy controls (Fig. 5). For all measurement points, the inter-examiner ICC for CT was 0.91 (95\% confidence interval 0.88-0.95). Subfoveal, nasal, and temporal CT were significantly higher in the active period of the disease ( $p<0.0001, p=0.028$, and $p=0.005$, respectively) (Fig. 2a, ). In contrast, choroidal vascularity was significantly lower in the active period of the disease $(p<0.0001)$ (Fig. 2b-d, ). In parallel with the decrease in choroidal vascularity, choroidal stroma and hence stromal/vascular area ratio increased significantly in the active period of the disease $(p<0.0001)$ (Fig. 2b-d, ). The characteristic changes of the choroid are detailed in Table 3. 


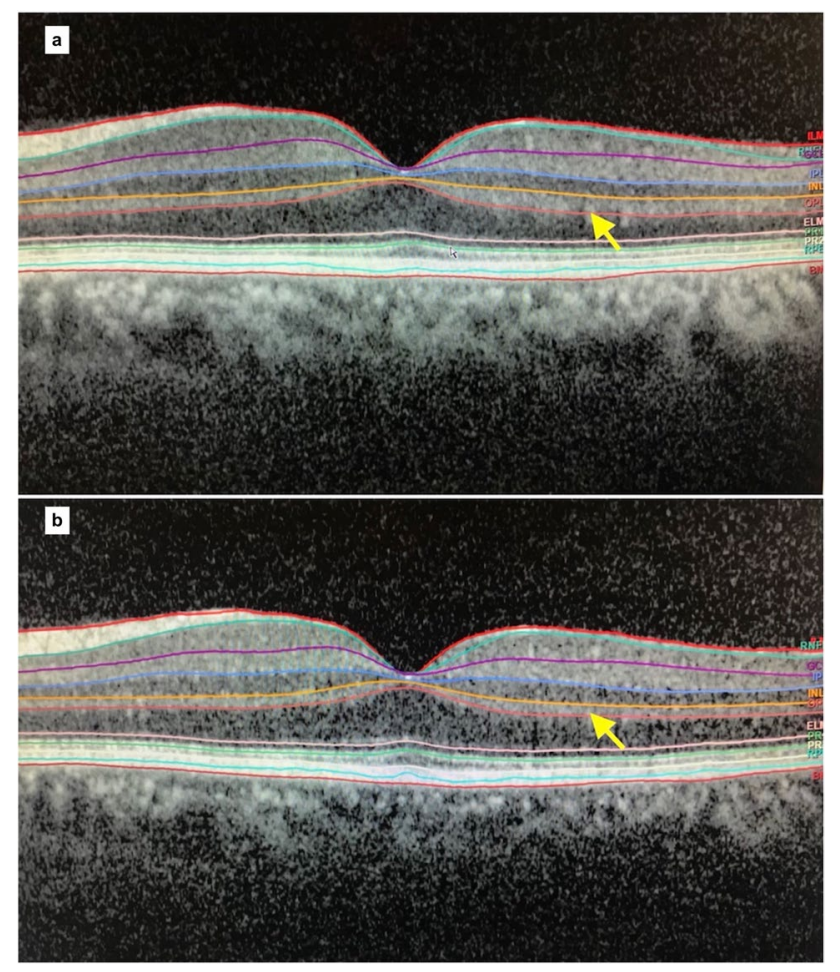

Fig. 1 Automatic segmentation of the macula by OCT software shows increased thickness of the outer plexiform layer in the active period of the disease (a, arrow) compared to after recovery (b, arrow)

Peripapillary RNFL thicknesses were significantly higher in all sectors in the active period of the disease, compared to after the recovery and healthy controls. The global mean value for all quadrants of peripapillary RNFL thicknesses significantly decreased after the recovery from $102.0 \pm 10.7$ to $98.3 \pm 7.7$ ( $p=0.001)$ (Fig. 3). Details of peripapillary RNFL thickness changes are showed in Table 4.

In the correlation analysis, a weak positive correlation was found between CRP levels and the mean differences in the CMT and CT $(r=0.403, p=0.001$, and $r=0.297$, $p=0.013$, respectively). There were no correlations among posterior ocular structure changes and WBC, ESR, procalcitonin, D-dimer, and $\mathrm{SpO} 2$ (for all, $p<0.05$ ).

The reflectivity of OCT echo of the choroid and peripapillary RNFL was significantly increased in the active period of the disease compared to after the recovery and healthy controls ( $p=0.023$ and $p<0.0001$, respectively) (Fig. 4). The analysis of the reflectivity of OCT echo of the posterior ocular structures is summarized in Table 5 . In the correlation analysis, a weak positive correlation was found between serum concentration levels of all acute phase reactants, including CRP, ESR, and procalcitonin, and the mean differences of the reflectivity of choroidal OCT echo ( $r=0.287, p=0.016, r=0.357, p=0.002$, and $r=0.245$, $p=0.041$, respectively). There were no correlations among the reflectivity of peripapillary RNFL and retinal layers and
WBC, CRP, ESR, procalcitonin, D-dimer, and $\mathrm{SpO} 2$ (for all, $p<0.05)$. Abnormal retinal findings were not observed in any patient on dilated fundus examination.

\section{Discussion}

The primary determinant of coronavirus for cellular infection is its spike in protein, which mediates the viral infection by binding to the cellular angiotensin-converting enzyme 2 (ACE2), with serine protease TMPRSS2 promoting spike protein priming. Recently, CD147-spike protein has been described as a novel route for SARSCoV-2 infection to host cells. Both the ACE2 and CD147 are highly expressed in human eyes, suggesting their potential role in ocular manifestations [10]. Indeed, SARSCoV-2 genomes were detected in human retinal biopsies of deceased COVID-19 patients [3]. In addition to these, endotheliitis and endothelial damage as a direct consequence of viral involvement and the host inflammatory response have recently been described as one of the most prominent causes of the systemic vascular thromboembolic and/or inflammatory manifestations of COVID-19 [11]. In previous studies, retinal hemorrhages, cotton wool retinal exudates, increased retinal vessel diameters, and tortuous vessels were reported in COVID-19 patients [4, 5]. Besides these, in an OCT angiography study, the superficial and deep retinal capillary plexus' vessel density was reported as significantly lower in patients recovered from COVID-19, versus age-matched normal controls [12].

This study demonstrated significant alterations in the outer plexiform layer, peripapillary RNFL, and choroidal structural, as well as vascular characteristics, in severe COVID-19 patients. These alterations may be associated directly with host-virus interaction or linked to an underlying autoimmune process inducing vasculitis or viral-mediated inflammation. In the present study, a weak correlation between CRP levels and the mean differences in the CMT and CT suggests the possibility of viral-mediated inflammation. A weak positive correlation between the choroidal reflectivity of OCT echoes and the concentration levels of acute phase reactants also supports this idea. However, there was no finding suggestive of uveitis in any patients of this study. Further studies that assess biochemical and histopathological data beyond ophthalmological aspects are warranted to better understand these issues.

Our results showed significantly higher OPL thickness without cystoid edema in the automated segmentation analysis, during the active period of the disease. This increase in thickness OPL could be due to intracellular edema of the Müller cell cytoplasm, which was most evident in the OPL of the foveal/macular region. These findings may be associated with disruption of the blood-retinal 

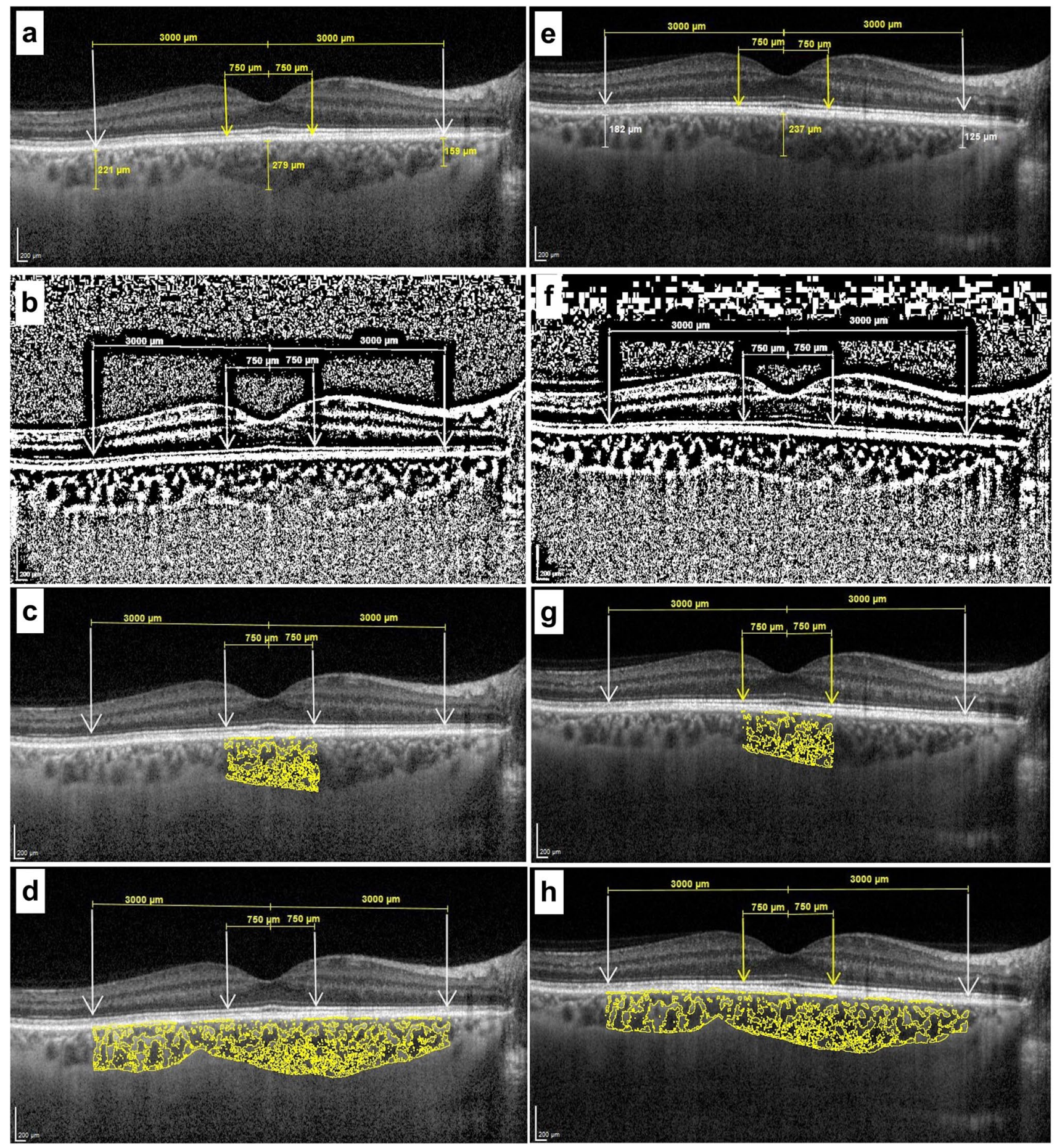

Fig. 2 Comparison of choroidal thickness, vascular, and stromal areas of the same eye in one of our patients in the active period of the disease $(\mathbf{a}, \mathbf{b}, \mathbf{c}, \mathbf{d})$ and after recovery $(\mathbf{e}, \mathbf{f}, \mathbf{g}, \mathbf{h})$. Measurement of choroidal thickness from the central subfoveal area and nasal and temporal $3000 \mu$ using the spectral domain optical coherence tomography (SD-OCT) software's caliper tool demonstrates thick choroid in the active period of the disease (a) compared to after recovery (e). Also, please note the choroid's hyperreflective echo in the active period of the disease (a) compared to after recovery (e). An OCT section after

binarization by ImageJ software using the Niblack auto local threshold technique $(\mathbf{b}, \mathbf{f})$. Black areas observed in the choroid represent the vascular area and white areas represent the stromal area. A superimposed image of the binarized segment over the EDI-OCT scan shows the optically segmented choroid into two parts, vascular and stromal areas $(\mathbf{c}, \mathbf{d}, \mathbf{g}, \mathbf{h})$. These areas were calculated separately for both the fovea $(\mathbf{c}, \mathbf{g})$ and the macula $(\mathbf{d}, \mathbf{h})$. Please note that the stromal area increases in both the fovea and the macula during the active phase of the disease 

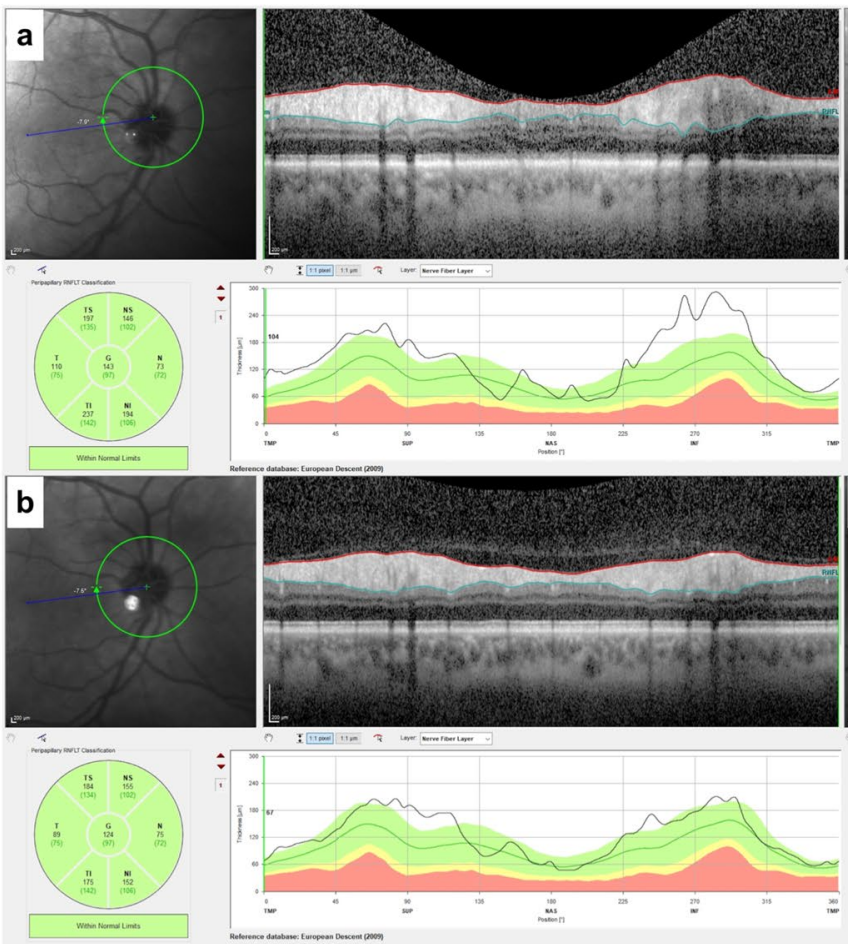
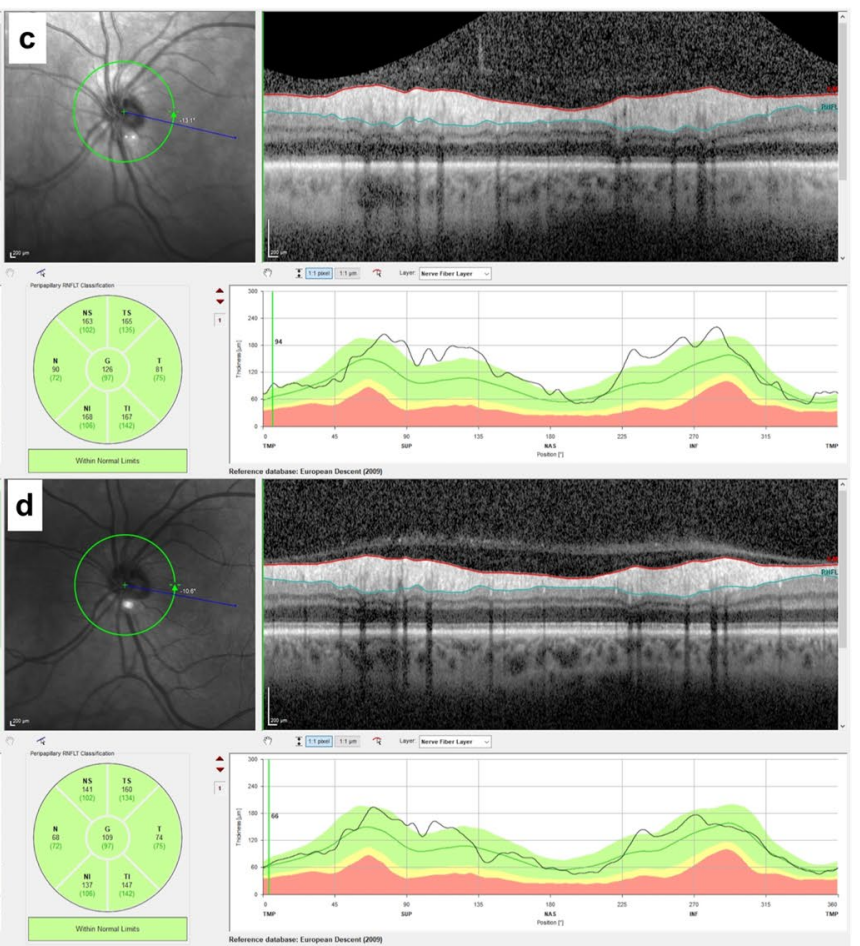

Fig. 3 The analysis of peripapillary retinal nerve fiber layer thickness in one of our patients shows an increase in all quadrants' thicknesses in both eyes during the active period of the disease $(\mathbf{a}, \mathbf{c})$ compared to after recovery $(\mathbf{b}, \mathbf{d})$

barrier, inflammation, or infection-mediated temporary retinal ischemia. Previous histologic studies have demonstrated that the OPL is mainly involved in cystoid spaces and contributes partly to macular thickening. Previous studies also suggested prolonged, excessive intracellular edema of Müller cells as the initial extracellular fluid collection process $[13,14]$. At the initial eye examination, all of our patients had 20/20 vision and had no visual loss symptoms, despite OPL changes.

In a recent study, the peripapillary vascular network was examined with structural OCT and OCT angiography in early post-COVID-19 patients [15]. The authors reported lower perfusion density of the radial peripapillary capillary plexus with a positive linear correlation of the RNFL average thickness in post-COVID-19 patients, compared to the healthy controls, which was attributed to the microvascular retinal impairment. Abrishami et al. [16] reported the higher peripapillary RNFL thicknesses in all sectors without statistical significance in patients with a history of COVID-19, at least 2 weeks after recovery. In our study, peripapillary RNFL thicknesses were significantly higher in all sectors in the active period of the disease, compared to the recovery and healthy controls (Table 4). These results may be related to the capture of measurements prior to the start of the patients' treatment in the active phase of the disease in our study: we know that steroids are used to suppress inflammation in the treatment of severe COVID-19 patients, and they can affect the peripapillary RNFL thickness. Our study findings clearly indicate optic nerve head involvement in severe COVID-19. Many infections and inflammatory processes of the central nervous system affect the optic nerve head. Since the neurologic manifestations are common with the SARS-CoV-2 infection [17], a potential optic nerve involvement should be kept in mind in managing COVID-19 patients.

New insights into a precise analysis of choroid pathologies using EDI-OCT have shown that choroidal structure and function are susceptible to many ocular and systemic diseases. The choroid can expand secondary to systemic or local inflammation $[18,19]$. In the present study, we found that CT (subfoveal, nasal, and temporal) and foveal and macular choroidal areas were significantly higher in the active period of the disease. Although we had to measure CT manually, inter-observer reproducibility was high in our study (for all manually measured variables, ICC was 0.91 ). The binarization of EDI-OCT images (Fig. 2b-d, ) demonstrates that the SARS-CoV-2 infection induces substantial edema of the choroidal stromal area, reflecting choroidal vasculitis, systemic inflammation, or hemodynamic impact of the respiratory disease. In addition to these findings, the choroidal reflectivity of OCT echo was increased in the active phase of the disease, with a weak positive correlation of 
Fig. 4 Representative macular, choroidal, and peripapillary retinal nerve fiber layer (RNFL) OCT images in a patient shows increasing grades of the reflectivity of OCT echo in the active period of the disease (a, $\mathbf{c}$, and e), compared to after recovery (b, d, and f). The corresponding mean values (bordered by the red rectangles) of the reflectivity of OCT echo are seen on the histogram (g-l) from the region of interest $(\mathbf{a}-\mathbf{f}$, bordered by the yellow lines) by image processing software (Image J)
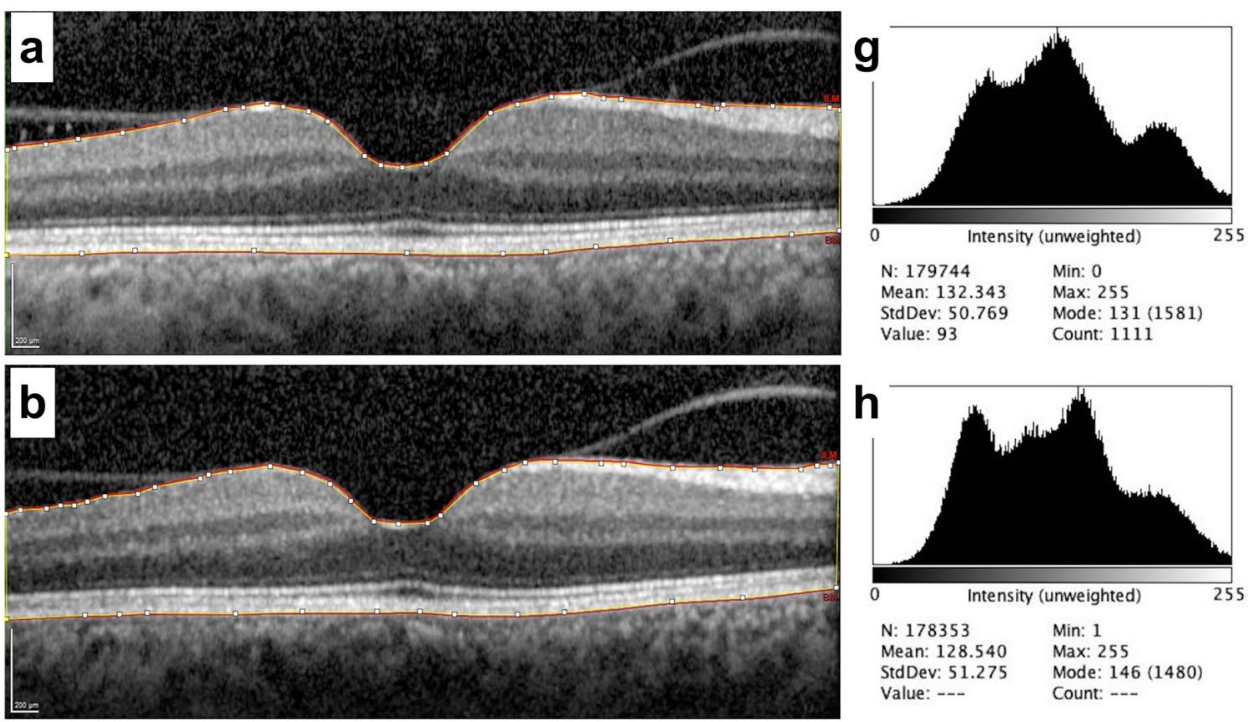

$$
\begin{array}{ll}
\text { N: } 179744 & \text { Min: } 0 \\
\text { Mean: } 132.343 & \text { Max: } 255 \\
\text { StdDev: } 50.769 & \text { Mode: } 131(1581) \\
\text { Value: } 93 & \text { Count: } 1111
\end{array}
$$

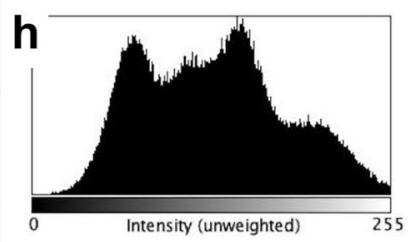

N: $178353 \quad$ Min: 1

StdDev: 51.275 Mode: $146(1480)$

Value: --- Count: -.-
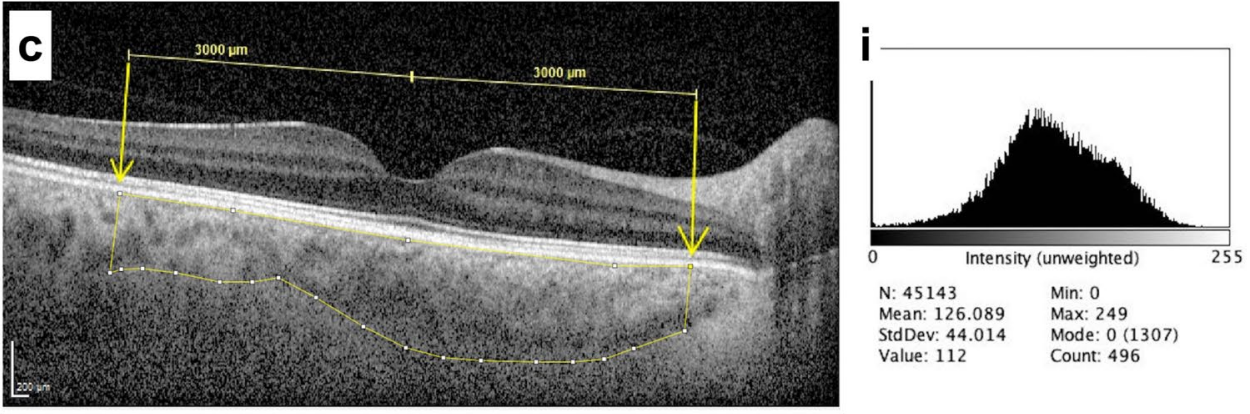

$\begin{array}{ll}\text { N: } 45143 & \text { Min: } 0 \\ \text { Mean: } 126.089 & \text { Max: } 249\end{array}$

StdDev: 44.014 Mode: 0 (1307)

Value: 112 Count: 496
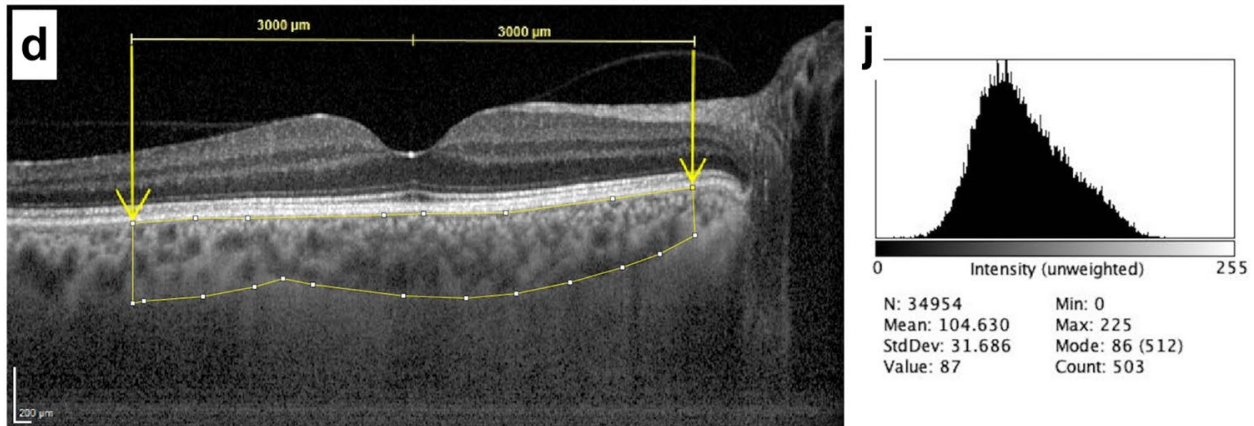

N: $34954 \quad$ Min: 0

Mean: 104.630 Max: 225

StdDev: 31.686 Mode: 86 (512)

Value: 87

Count: 503
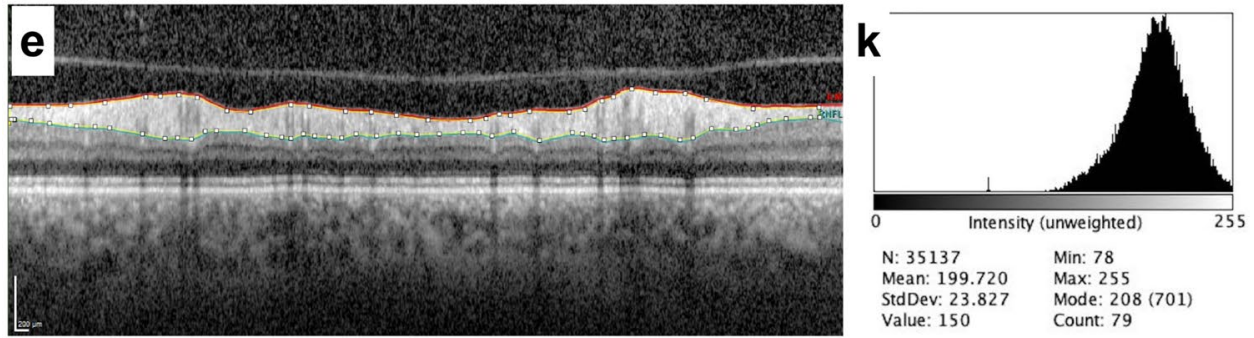

N: $35137 \quad$ Min: 78

Mean: 199.720 Max: 255

StdDev: 23.827 Mode: 208 (701) Value: $150 \quad$ Count: 79

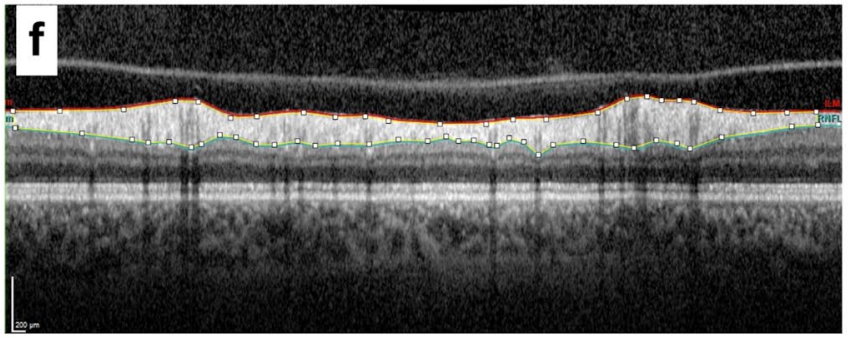


Table 2 Automatic measurements and comparisons of inner and outer retinal layers of the macula by SD-OCT software at hospital admission and after recovery, compared with age- and gender-matched healthy controls

\begin{tabular}{lcccc}
\hline Retinal layers characteristics & $\begin{array}{c}\text { At hospital admis- } \\
\text { sion }(\text { mean } \pm \text { SD) }\end{array}$ & $\begin{array}{l}\text { After recovery } \\
(\text { mean } \pm \text { SD) }\end{array}$ & $\begin{array}{l}\text { Healthy controls } \\
(\text { mean } \pm \text { SD) }\end{array}$ & $p$ value* $^{*}$ \\
\hline Macula OCT images' quality scores & $24.1 \pm 2.8$ & $24.3 \pm 2.7$ & $24.5 \pm 2.9$ & 0.679 \\
Central macular thickness, $\mu \mathrm{m}$ & $276.2 \pm 24.0$ & $272.4 \pm 23.5$ & $269.8 \pm 24.6$ & 0.285 \\
Retina nerve fiber layer, $\mu \mathrm{m}$ & $12.9 \pm 2.6$ & $12.8 \pm 2.6$ & $12.8 \pm 2.8$ & 0.798 \\
Ganglion cell layer, $\mu \mathrm{m}$ & $16.5 \pm 5.4$ & $16.4 \pm 5.2$ & $16.6 \pm 8.3$ & 0.983 \\
Inner plexiform layer, $\mu \mathrm{m}$ & $21.4 \pm 4.9$ & $21.1 \pm 4.4$ & $21.1 \pm 5.0$ & 0.933 \\
Inner nuclear layer, $\mu \mathrm{m}$ & $22.6 \pm 5.6$ & $22.6 \pm 8.1$ & $22.4 \pm 6.7$ & 0.970 \\
Outer plexiform layer, $\mu \mathrm{m}$ & $29.6 \pm 8.8$ & $26.5 \pm 5.8$ & $25.8 \pm 6.3$ & 0.004 \\
Outer nuclear layer, $\mu \mathrm{m}$ & $86.9 \pm 12.3$ & $86.6 \pm 9.1$ & $85.7 \pm 11.4$ & 0.791 \\
Retinal pigment epithelium, $\mu \mathrm{m}$ & $16.6 \pm 1.4$ & $16.7 \pm 1.5$ & $16.7 \pm 2.9$ & 0.979 \\
Inner retinal layer, $\mu \mathrm{m}$ & $189.3 \pm 23.6$ & $184.9 \pm 22.2$ & $184.2 \pm 23.2$ & 0.366 \\
Outer retinal layer, $\mu \mathrm{m}$ & $86.7 \pm 3.7$ & $86.6 \pm 5.1$ & $85.7 \pm 3.8$ & 0.286 \\
\hline
\end{tabular}

${ }^{¥}$ At the third month of follow-up

*One-way analysis of variance (statistically significant $p$ values is shown in italic font)

Table 3 Choroidal characteristics of studied patients at hospital admission and after recovery, compared with age- and gender-matched healthy controls

\begin{tabular}{|c|c|c|c|c|}
\hline Choroidal characteristics & $\begin{array}{l}\text { At hospital admis- } \\
\text { sion }(\text { mean } \pm S D)\end{array}$ & After recovery $^{\ddagger}($ mean $\pm S D)$ & Healthy controls $($ mean \pm SD) & $p$ value* \\
\hline EDI-OCT images' quality scores & $23.4 \pm 2.5$ & $23.7 \pm 2.3$ & $23.6 \pm 2.6$ & 0.716 \\
\hline Subfoveal choroidal thickness, $\mu \mathrm{m}$ & $352.6 \pm 67.3$ & $317.8 \pm 66.1$ & $314.0 \pm 41.0$ & $<0.0001$ \\
\hline Nasal $3 \mathrm{~mm}$ choroidal thickness, $\mu \mathrm{m}$ & $202.2 \pm 55.2$ & $183.2 \pm 57.6$ & $178.1 \pm 54.1$ & 0.028 \\
\hline Temporal $3 \mathrm{~mm}$ choroidal thickness, $\mu \mathrm{m}$ & $252.9 \pm 59.8$ & $227.7 \pm 57.5$ & $231.4 \pm 15.2$ & 0.005 \\
\hline Mean choroidal thickness, $\mu \mathrm{m}$ & $269.2 \pm 49.7$ & $242.9 \pm 48.8$ & $241.2 \pm 24.8$ & $<0.0001$ \\
\hline \multicolumn{5}{|l|}{ Subfoveal (1.5 mm region) } \\
\hline Total choroidal area, $\mathrm{mm}^{2}$ & $0.56 \pm 0.11$ & $0.50 \pm 0.09$ & $0.49 \pm 0.10$ & $<0.0001$ \\
\hline Vascular area, $\mathrm{mm}^{2}$ & $0.34 \pm 0.06$ & $0.32 \pm 0.06$ & $0.31 \pm 0.07$ & 0.066 \\
\hline Stromal area, $\mathrm{mm}^{2}$ & $0.22 \pm 0.06$ & $0.18 \pm 0.03$ & $0.18 \pm 0.03$ & $<0.0001$ \\
\hline Stromal/vascular area ratio & $0.65 \pm 0.08$ & $0.58 \pm 0.06$ & $0.57 \pm 0.07$ & $<0.0001$ \\
\hline Choroidal vascularity index & $0.61 \pm 0.02$ & $0.63 \pm 0.02$ & $0.64 \pm 0.03$ & $<0.0001$ \\
\hline \multicolumn{5}{|l|}{ Macular (6 mm region) } \\
\hline Total choroidal area, $\mathrm{mm}^{2}$ & $1.79 \pm 0.32$ & $1.64 \pm 0.30$ & $1.63 \pm 0.28$ & 0.002 \\
\hline Vascular area, $\mathrm{mm}^{2}$ & $1.07 \pm 0.15$ & $1.04 \pm 0.21$ & $1.04 \pm 0.20$ & 0.443 \\
\hline Stromal area, $\mathrm{mm}^{2}$ & $0.72 \pm 0.17$ & $0.60 \pm 0.10$ & $0.59 \pm 0.08$ & $<0.0001$ \\
\hline Stromal/vascular area ratio & $0.66 \pm 0.08$ & $0.59 \pm 0.06$ & $0.58 \pm 0.07$ & $<0.0001$ \\
\hline Choroidal vascularity index & $0.60 \pm 0.03$ & $0.63 \pm 0.02$ & $0.63 \pm 0.03$ & $<0.0001$ \\
\hline
\end{tabular}

${ }^{¥}$ At the third month of follow-up

*One-way analysis of variance (statistically significant $p$ values are shown in italic font)

the concentration levels of acute phase reactants (Fig. 4 and Table 5). CVI, which corresponds to the choroidal vascular area ratio to the total choroidal area, has been a novel tool to evaluate the choroidal vascular status: a decreased CVI was reported in inflammatory disorders [20]. Our study showed that choroidal vascularity was significantly lower and that the stromal area to vascular area $(\mathrm{S} / \mathrm{V})$ ratio of the choroid was significantly higher, in the active period of the disease. These results could be associated with choroidal stromal edema (Fig. 2b-d, ) as well as narrowing of the choroidal vessel lumens, due to choroidal endothelial cell inflammation and choroidal vessel wall thickening. Besides all these, micro-thromboembolic complications of the choroidal vessels may have contributed to this result. In a recent study, the disease's thrombotic complications were reported in hospitalized COVID-19 patients varying from 12 to $21 \%$ [21].

OCT reflectivity provides a detailed, quantitative description of the specialized regions, including retinal, choroidal, 
Table 4 Automatic

measurements and comparisons of peripapillary retinal nerve fiber layer (RNFL) by SD-OCT software at hospital admission and after recovery, compared with age- and gender-matched healthy controls

\begin{tabular}{lcccc}
\hline Peripapillary RNFL & $\begin{array}{l}\text { At hospital admis- } \\
\text { sion }(\text { mean } \pm \text { SD) }\end{array}$ & $\begin{array}{l}\text { After recovery } \\
(\text { mean } \pm \text { SD })\end{array}$ & $\begin{array}{l}\text { Healthy control } \\
(\text { mean } \pm \text { SD) }\end{array}$ & $p$ value* $^{*}$ \\
\hline RNFL images' quality scores & $25.2 \pm 2.7$ & $25.4 \pm 2.5$ & $25.4 \pm 2.7$ & $0.791^{\diamond}$ \\
Global $\left(360^{\circ}, \mu \mathrm{m}\right)$ & $102.0 \pm 10.7$ & $98.3 \pm 7.7$ & $97.2 \pm 4.4$ & $0.001^{\Delta}$ \\
Temporal $\left(315-45^{\circ}, \mu \mathrm{m}\right)$ & $78.8 \pm 12.7$ & $74.0 \pm 10.3$ & $73.9 \pm 7.8$ & $0.008^{\diamond}$ \\
Temporal-superior $\left(45-90^{\circ}, \mu \mathrm{m}\right)$ & $139.5 \pm 22.1$ & $132.3 \pm 18.9$ & $130.4 \pm 18.4$ & $0.014^{\Delta}$ \\
Temporal-inferior $\left(270-315^{\circ}, \mu \mathrm{m}\right)$ & $147.2 \pm 22.1$ & $141.1 \pm 13.9$ & $139.3 \pm 15.4$ & $0.021^{\diamond}$ \\
Nasal $\left(135-225^{\circ}, \mu \mathrm{m}\right)$ & $73.6 \pm 12.0$ & $71.9 \pm 9.3$ & $68.6 \pm 12.2$ & $0.029^{\diamond}$ \\
Nasal-superior $\left(90-135^{\circ}, \mu \mathrm{m}\right)$ & $110.9 \pm 22.0$ & $103.3 \pm 19.0$ & $103.5 \pm 15.8$ & $0.006^{\Delta}$ \\
Nasal-inferior $\left(225-270^{\circ}, \mu \mathrm{m}\right)$ & $114.8 \pm 28.2$ & $106.9 \pm 19.7$ & $106.2 \pm 10.6$ & $0.027^{\diamond}$ \\
\hline
\end{tabular}

${ }^{¥}$ At the third month of follow-up

${ }^{\Delta}$ Kruskal-Wallis test

$\diamond_{\text {One-way analysis of variance }}$
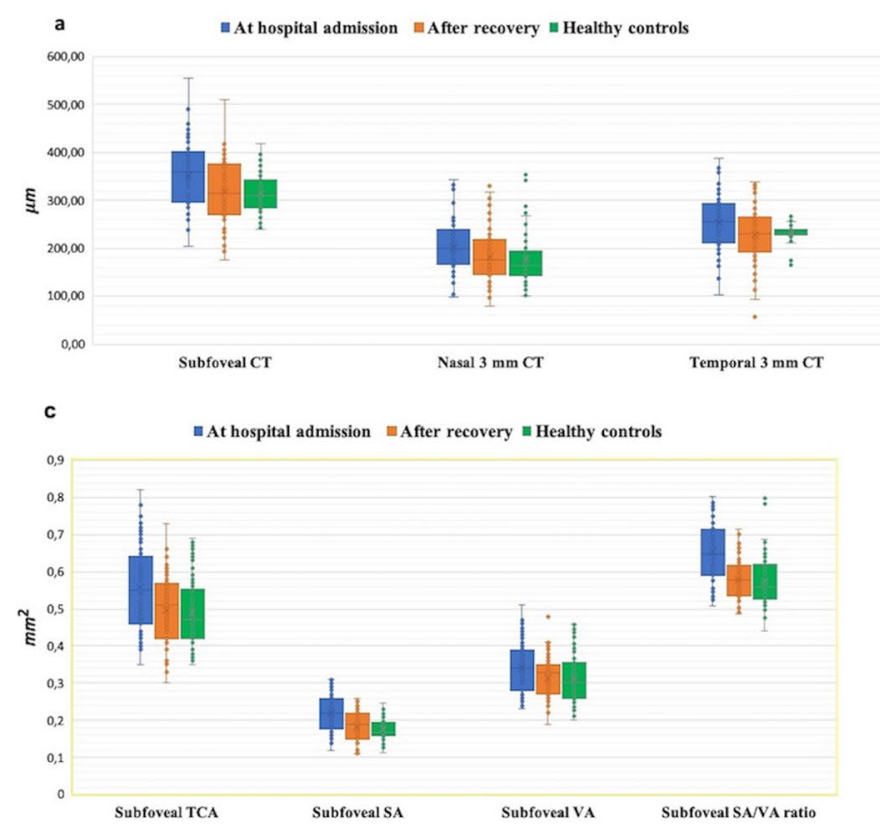

Fig. 5 Composite box plots representing the measurements at hospital admission, compared to after recovery and age- and gendermatched healthy controls for a choroidal thickness (CT), b choroidal vascularity index (CVI), c subfoveal total choroidal area (TCA), subfoveal stromal area (SA), subfoveal vascular area (VA) and subfoveal

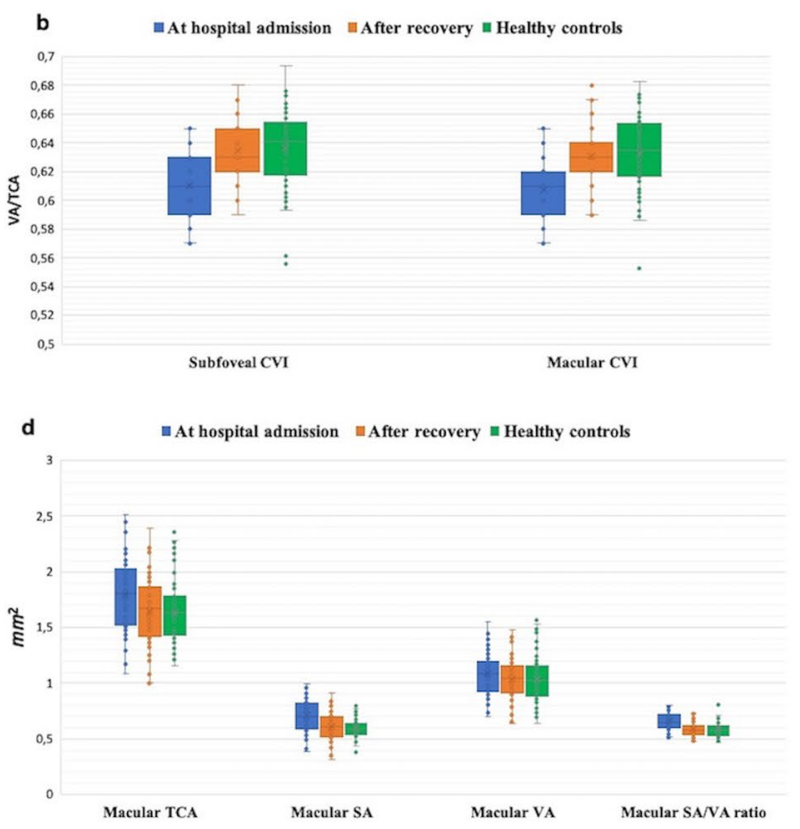

stromal/vascular area (SA/VA) ratio, (d) macular TCA, macular SA, macular VA, and macular SA/VA ratio. Please note that all measurements showed statistically significant changes at hospital admission, compared to after recovery and healthy controls except for the subfoveal and macular vascular area (for all, $p<0.05$ )
Table 5 The reflectivity of OCT echoes measured by ImageJ software at hospital admission compared with after recovery and age- and gender-matched healthy controls

\begin{tabular}{llccr}
\hline The reflectivity of OCT echo & $\begin{array}{l}\text { At hospital admis- } \\
\text { sion }(\text { mean } \pm \text { SD) }\end{array}$ & $\begin{array}{l}\text { After recovery } \\
(\text { mean } \pm \text { SD) }\end{array}$ & $\begin{array}{l}\text { Healthy controls } \\
(\text { mean } \pm \text { SD })\end{array}$ & $p$ value* $^{*}$ \\
\hline Retinal layers reflectivity & $123.3 \pm 10.7$ & $120.2 \pm 7.0$ & $120.3 \pm 8.3$ & 0.057 \\
Choroidal reflectivity & $103.0 \pm 16.8$ & $97.8 \pm 17.1$ & $96.0 \pm 12.4$ & 0.023 \\
Peripapillary RNFL reflectivity & $183.3 \pm 8.6$ & $178.2 \pm 8.9$ & $176.7 .4 \pm 7.2$ & $<0.0001$ \\
\hline
\end{tabular}

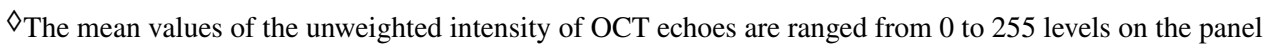

${ }^{¥}$ At the third month of follow-up

* One-way analysis of variance 
and peripapillary RNFL structures. One of the important results of this study was the increase in the reflectivity of OCT echo in the choroid and peripapillary RNFL during the active period of the disease, compared to after recovery and healthy controls (Fig. 4 and Table 5). Considering that the choroidal stromal area is hyperreflective and the vascular structure is hyporeflective in EDI-OCT, an increase in reflectivity of OCT echo of the choroidal structure means that the vascular area in the choroid is decreased or the stromal area is increased, or that both conditions develop together. Increased peripapillary RNFL thickness and reflectivity in the acute phase of severe COVID-19 suggest COVID19-related optic nerve head involvement due to infection or inflammation.

One important point is that all of the ocular structural changes presented above developed in previously healthy individuals who were included in this study. Indeed, we do not know the effect of these changes in patients with active retinal pathologies such as diabetic retinopathy, retinal vein occlusion, and age-related macular degeneration. Further investigations are needed on this subject.

Marinho et al. [22] reported abnormal retinal findings in 12 patients with COVID-19 and illustrated OCT findings of three patients with hyperreflective bands in the inner retina. However, these OCT findings significantly mimic normal inner retinal vessels, which Vavvas et al. [23] interpreted as normal retinal vessels. Indeed, we observed some hyperreflective lesions in the same retinal layers as reported by Marinho et al., and we also think these hyperreflective bands are probably normal retinal vessels. We did not find abnormal retinal findings in any patient of this study.

One strength of the present study is to have explored posterior segment involvement in severe COVID-19 pneumonia before any treatment has begun, to avoid potential bias resulting from systemic anti-inflammatory medications, and in patients who had no comorbidities at the time of their hospitalization. One of the shortcomings of this study is that the other visual function components, such as visual field, contrast sensitivity, color vision, dark adaptation, and stereoscopic vision other than the BCVA, were not investigated. Another shortcoming of this study is that intubated patients or patients followed in the intensive care unit were not included in the study.

In conclusion, this study detected significant posterior ocular structure alterations in severe COVID-19 patients without abnormal retinal examination findings. In the active period of the disease, the OPL increased, average CT and choroidal area were significantly higher, choroidal vascularity decreased, choroidal stromal area increased instead of the vascular area, peripapillary RNFL thicknesses were significantly higher in all sectors, and the reflectivity of the OCT echo of the choroid and peripapillary RNFL increased. These findings may be associated with direct host-virus interaction, or linked to an autoimmune process, vasculopathy, or still yet of a viral-mediated inflammation.

\section{Declarations}

Ethics approval All procedures were performed following the ethical standards of the University of Health Sciences, Kayseri City Training Research Hospital, and the 1964 Helsinki declaration and its later amendments. The study protocol was approved by the Institutional Review Board (\#135719921).

Informed consent Informed consent was obtained from all individual participants included in the study.

Conflict of interest The authors declare no competing interests.

\section{References}

1. Siddiqi HK, Libby P, Ridker PM (2021) COVID-19 - a vascular disease. Trends Cardiovasc Med 31:1-5. https://doi.org/10.1016/j. tcm.2020.10.005

2. Giamarellos-Bourboulis EJ, Netea MG, Rovina N et al (2020) Complex immune dysregulation in COVID-19 patients with severe respiratory failure. Cell Host Microbe 27:992-1000.e3. https://doi.org/10.1016/j.chom.2020.04.009

3. Casagrande M, Fitzek A, Püschel K, Aleshcheva G, Schultheiss HP, Berneking L, Spitzer MS, Schultheiss M (2020) Detection of SARS-CoV-2 in Human Retinal Biopsies of Deceased COVID-19 Patients. Ocul Immunol Inflamm 28:721-725. https://doi.org/10. 1080/09273948.2020.1770301

4. Landecho MF, Yuste JR, Gándara E, Sunsundegui P, Quiroga J, Alcaide AB, García-Layana A (2021) COVID-19 retinal microangiopathy as an in vivo biomarker of systemic vascular disease? J Intern Med 289:116-120. https://doi.org/10.1111/joim.13156

5. Invernizzi A, Torre A, Parrulli S et al (2020) Retinal findings in patients with COVID-19: Results from the SERPICO-19 study. EClinicalMedicine 27:100550. https://doi.org/10.1016/j.eclinm. 2020.100550

6. Steiner M, Esteban-Ortega MDM, Muñoz-Fernández S (2019) Choroidal and retinal thickness in systemic autoimmune and inflammatory diseases: a review. Surv Ophthalmol 64:757-769. https://doi.org/10.1016/j.survophthal.2019.04.007

7. Wu Z, McGoogan JM (2020) Characteristics of and important lessons from the coronavirus disease 2019 (COVID-19) outbreak in China: summary of a report of 72314 cases from the Chinese Center for Disease Control and Prevention. JAMA 323:12391242. https://doi.org/10.1001/jama.2020.2648

8. American Academy of Ophthalmology (2020) Important coronavirus updates for ophthalmologists. Available at https://www. aao.org/headline/alert-important-coronavirus-context\#resuming Accessed 3 June 2021

9. Agrawal R, Gupta P, Tan KA, Cheung CM, Wong TY, Cheng CY (2016) Choroidal vascularity index as a measure of vascular status of the choroid: measurements in healthy eyes from a populationbased study. Sci Rep 6:21090. https://doi.org/10.1038/srep21090

10. Li YP, Ma Y, Wang N, Jin ZB (2021) Eyes on coronavirus. Stem Cell Res 51:102200. https://doi.org/10.1016/j.scr.2021.102200

11. Varga Z, Flammer AJ, Steiger P, Haberecker M, Andermatt R, Zinkernagel AS, Mehra MR, Schuepbach RA, Ruschitzka F, Moch H (2020) Endothelial cell infection and endotheliitis in 
COVID-19. Lancet 395(10234):1417-1418. https://doi.org/10. 1016/S0140-6736(20)30937-5

12. Abrishami M, Emamverdian Z, Shoeibi N, Omidtabrizi A, Daneshvar R, Saeidi Rezvani T, Saeedian N, Eslami S, Mazloumi M, Sadda S, Sarraf D (2021) Optical coherence tomography angiography analysis of the retina in patients recovered from COVID19: a case-control study. Can J Ophthalmol 56:24-30. https://doi. org/10.1016/j.jcjo.2020.11.006

13. Yanoff M, Fine BS, Brucker AJ, Eagle RC Jr (1984) Pathology of human cystoid macular edema. Surv Ophthalmol 28(Suppl):505511. https://doi.org/10.1016/0039-6257(84)90233-9

14. Tso MO (1982) Pathology of cystoid macular edema. Ophthalmology 89:902-915. https://doi.org/10.1016/s0161-6420(82)34698-9

15. Savastano A, Crincoli E, Savastano MC, Younis S, Gambini G, De Vico U, Cozzupoli GM, Culiersi C, Rizzo S, Gemelli Against Covid-Post-Acute Care Study Group (2020) Peripapillary Retinal Vascular Involvement in Early Post-COVID-19 Patients. J Clin Med 9:2895. https://doi.org/10.3390/jcm9092895

16. Abrishami M, Daneshvar R, Emamverdian Z, Tohidinezhad F, Eslami S (2021) optic nerve head parameters and peripapillary retinal nerve fiber layer thickness in patients with coronavirus disease 2019. Ocul Immunol Inflamm 19:1-4. https://doi.org/10. 1080/09273948.2020.1850800

17. Correia AO, Feitosa PWG, Moreira JLS, Nogueira SÁR, Fonseca RB, Nobre MEP (2020) Neurological manifestations of COVID19 and other coronaviruses: a systematic review. Neurol Psychiatry Brain Res 37:27-32. https://doi.org/10.1016/j.npbr.2020.05. 008

18. Kim M, Kim H, Kwon HJ, Kim SS, Koh HJ, Lee SC (2013) Choroidal thickness in Behcet's uveitis: an enhanced depth imaging-optical coherence tomography and its association with angiographic changes. Invest Ophthalmol Vis Sci 54:6033-6039. https://doi.org/10.1167/iovs.13-12231

19. Baytaroğlu A, Kadayifçilar S, Ağin A, Deliktaş Ö, Demir S, Bilginer Y, Karakaya J, Özen S, Eldem B (2020) Choroidal vascularity index as a biomarker of systemic inflammation in childhood Polyarteritis Nodosa and adenosine deaminase-2 deficiency. Pediatr Rheumatol Online J 18:29. https://doi.org/10.1186/ s12969-020-0417-3

20. Agrawal R, Salman M, Tan KA, Karampelas M, Sim DA, Keane PA, Pavesio C (2016) Choroidal Vascularity Index (CVI)-a novel optical coherence tomography parameter for monitoring patients with panuveitis? PLoS ONE 11:e0146344. https://doi.org/10. 1371/journal.pone.0146344

21. Dutch COVID, Coalition T, Kaptein FHJ, Stals MAM et al (2021) Incidence of thrombotic complications and overall survival in hospitalized patients with COVID-19 in the second and first wave. Thromb Res 199:143-148. https://doi.org/10.1016/j.thromres. 2020.12.019

22. Marinho PM, Marcos AAA, Romano AC, Nascimento H, Belfort $\mathrm{R} \mathrm{Jr}$ (2020) Retinal findings in patients with COVID-19. Lancet 395:1610. https://doi.org/10.1016/S0140-6736(20)31014-X

23. Vavvas DG, Sarraf D, Sadda SR, Eliott D, Ehlers JP, Waheed NK, Morizane Y, Sakamoto T, Tsilimbaris M, Miller JB (2020) Concerns about the interpretation of OCT and fundus findings in COVID-19 patients in recent Lancet publication. Eye (Lond) 34:2153-2154. https://doi.org/10.1038/s41433-020-1084-9

Publisher's note Springer Nature remains neutral with regard to jurisdictional claims in published maps and institutional affiliations. 